\title{
Milimetre Altı Ölçümler İçin Fiber Optik Yer Değiştirme Sensörü
}

\author{
Şekip Esat Hayber ${ }^{1}$, Timuçin Emre Tabaru ${ }^{2 *}$ \\ ${ }^{1}$ Kırşehir Ahi Evran Üniversitesi, Mühendislik Mimarlık Fakültesi, Elektrik Elektronik Mühendisliği Bölümü, Kırşehir, Türkiye, (ORCID: 0000-0003-0062-3817), \\ sehayber@ahievran.edu.tr \\ 2* Erciyes Üniversitesi, Klinik Mühendisliği Araştırma ve Uygulama Merkezi, Kayseri, Türkiye (ORCID: 0000-0002-1373-3620), etabaru@erciyes.edu.tr
}

(2nd International Conference on Access to Recent Advances in Engineering and Digitalization (ARACONF)-10-12 March 2021)

(DOI: 10.31590 /ejosat.901075)

ATIF/REFERENCE: Hayber, Ş. E. \& Tabaru, T. E. (2021). Milimetre Altı Ölçümler İçin Fiber Optik Yer Değiştirme Sensörü. Avrupa Bilim ve Teknoloji Dergisi, (24), 1-4.

$\ddot{O} \mathbf{z}$

Bu çalışma milimetre altı yer değiştirmenin algılanması için birden fazla fiber kayıp mekanizmasının aynı anda değerlendirilip sensör parametrelerinin elde edilmesine dayanmaktadır. Bu kapsamda fiber hizalama kayıplarından olan boyuna yanlış hizalama kayıplarına ilaveten Fresnel yansıma kayıplarının da birlikte değerlendirildiği bir sensör sistemi incelenmiştir. Her iki kayıp mekanizmasının etkisi tek bir eşitlik ile ifade edilip sensör sisteminde kullanılacak fiber tiplerinin etkileri araştırılmıştır. Böylelikle plastik optik fiberle oluşturulacak yer değiştirme sensörünün milimetre altı ölçüm yapabilmesinin incelenen fiber parametrelerinde en uygun değerler belirlenmiştir. Hesaplamalar sonucunda birbirinden farklı çap değerlerine sahip olan fiber tipleri için 1 mm'nin altında yer değiştirme ölçümlerinde kullanılacak en uygun fiber tipinin mümkün olan en düşük çapa sahip olması sonucuna ulaşılmıştır. Fiberlerin tipik çap değerleri dikkate alındığında bu durum için en uygun adayın 980/1000 (öz/yelek) $\mu \mathrm{m}$ çapa sahip olan fiber olduğu belirlenmiştir. Bu fiberin hassasiyetlik derecesi giriş fiberine uygulanan güç $100 \mu \mathrm{W}$ olduğunda $32,05 \mu \mathrm{W} / \mathrm{mm}$ olarak bulunmuştur. Bu sonuçlar $1960 \mu \mathrm{m}$ ve $2944 \mu \mathrm{m}$ öz çapına sahip olan diğer fiberler için sırasıyla, $16,02 \mu \mathrm{m} / \mathrm{mm}$ ve $10,67 \mu \mathrm{m} / \mathrm{mm}$ olarak bulunmuştur.

Anahtar Kelimeler: Fiber hizalama kayıpları, Fresnel yansıma kaybı, PMMA fiber optik sensör, yer değiştirme sensörü.

\section{Fiber Optic Displacement Sensor for Sub-Millimeter Measurements}

\begin{abstract}
This study is based on evaluating more than one fiber loss mechanism simultaneously to detect sub-millimeter displacement and obtaining sensor parameters. In this context, a sensor system that evaluates Fresnel reflection losses together in addition to longitudinal misalignment losses from fiber alignment losses was examined. The effects of both loss mechanisms are expressed in a single equation. The effects of the fiber types used in the sensor system have been investigated. Thus, the most suitable values were determined in the fiber parameters that the displacement sensor to be formed with plastic optic fiber can make a sub-millimeter measurement. As a result of the calculations, it has been concluded that the most suitable fiber type to be used in displacement measurements below 1 mm for fiber types with different diameter values has the lowest possible diameter. The fibers' typical diameter values have determined that the most suitable candidate for this situation is the fiber with a diameter of 980/1000 (core/vest) $\mu \mathrm{m}$. This fiber's sensitivity was found to be $32.05 \mu \mathrm{W} / \mathrm{mm}$ when the power applied to the input fiber was $100 \mu \mathrm{W}$. These results were found to be $16.02 \mu \mathrm{m} / \mathrm{mm}$ and 10.67 $\mu \mathrm{m} / \mathrm{mm}$ for other fibers with $1960 \mu \mathrm{m}$ and $2944 \mu \mathrm{m}$ core diameter, respectively.
\end{abstract}

Keywords: Fiber alignment losses, Fresnel reflection loss, PMMA fiber optic sensor, displacement sensor.

*Sorumlu Yazar: etabaru@erciyes.edu.tr 


\section{Giriş}

Mesafe ölçümleri için çeşitli optik sensörler literatürde rapor edilmiştir [1]. Bir konum sensörü, doğrusal hareket veya dönme açısından mutlak konumu (konum) veya göreceli konumu (yer değiştirme) gösterebilir. Yer değiştirme sensörü, çeşitli elemanlar boyunca yer değiştirme miktarını algılayarak ve bunu bir mesafeye dönüştürerek sensör ile bir nesne arasındaki mesafeyi ölçen bir cihazdır. Hangi elemanın kullanıldığına bağlı olarak, optik yer değiştirme sensörleri, doğrusal yakınlık sensörleri ve ultrasonik yer değiştirme sensörleri gibi çeşitli sensör türleri vardır. Temassız yer değiştirme sensörleri, çok sayıda endüstriyel uygulamaların önemli bir parçasını oluşturur. Bu nedenle, iyi performans seviyelerine sahip fakat düşük maliyetli ve pratik optik sensörler tercih sebebi olmuşlardır [2]. Fiber optik sensörler genel olarak yüksek çözünürlük sunan interferometrik [3] ve kısmen daha basit genlik modülasyonu [4] sensörler olmak üzere sınıflandırılabilirler. Fiber optik sensörler, avantajlı özellikleri sayesinde interferometrik optik sensörlere değerli bir alternatif oluşturur: yanmaz özelliklerinin yanı sıra elektromanyetik parazitlere karşı bağışıklık, doğası gereği yüksek hassasiyet, hafif, küçük boyut, hızlı tepki ve yüksek hassasiyetli temassız ölçüm ve uzaktan çalıştırma yetenekleri fiber optik sensörlerin kullanım sahasının genişliği artırır [5, 6]. Literatürde fiber optik tabanlı çok sayıda yer değiştirme sensörü geliştirilmiştir. Bunlardan FabryPerot interferometresine dayanan yapılar [7-9], sıvı refraktometre tabanlı [10], çok modlu girişim tabanlı [11], sicaklık ölçümü için yer değiştirmeyi kullanan yapılar [12], genlik modülasyonlu tek modlu fiber kullanan yapılar [13] olmak üzere sayılabilir. Fiber kayıp mekanizmalarıyla sensörler geliştirirken bazı durumlarda birden fazla mekanizmanın devrede olduğu durumla karşılaşılabilir. Örneğin yer değiştirme hizalama kayıplarına dayanan bir yer değiştirme sensöründe aynı zamanda yansıma kayıplarının da etkisini dahil etmek gerekmektedir. Her iki kayıp mekanizmasının etkilerinin bir arada değerlendirilerek oluşturulmuş olan bir yer değiştirme sensörüne literatürde rastlanmamıştır.

$\mathrm{Bu}$ çalışmada fiber hizalama kayıplarından olan boyuna yanlış hizalama kayıplarına ilaveten Fresnel yansıma kayıplarının da birlikte değerlendirildiği bir sensör sistemi incelenmiştir. Sistemin matematiksel eşitliğinden yola çıkarak tipik plastik optik fiber parametrelerinin sensör performansı üzerine etkileri araştırılmıştır. Böylelikle plastik optik fiberle oluşturulacak yer değiştirme sensörünün milimetre altı ölçüm yapabilmesinin gerek ve yeter koşulları üzerine odaklanılmıştır. Bu doğrultuda farklı çap değerlerine sahip olan fiber tipleri için $1 \mathrm{~mm}$ 'nin altında yer değiştirme ölçümlerinde kullanılacak en uygun fiber tipinin mümkün olan en düşük çapa sahip olması sonucuna ulaşılmıştır. Piyasadaki halihazırdaki çap değerleri dikkate alındığında bu durum için en uygun adayın 980/1000 (öz/yelek) $\mu$ m çapa sahip olan fiber olduğu belirlenmiştir. Bu fiberin hassasiyetlik derecesi giriş fiberine uygulanan güç $100 \mu \mathrm{W}$ olduğunda $32,05 \mu \mathrm{W} / \mathrm{mm}$ olarak bulunmuştur. Bu sonuçlar $1960 \mu \mathrm{m}$ ve $2944 \mu \mathrm{m}$ öz çapına sahip olan diğer fiberler için sirasıyla, $16,02 \mu \mathrm{m} / \mathrm{mm}$ ve 10,67 $\mu \mathrm{m} / \mathrm{mm}$ olarak bulunmuştur.

\section{Materyal ve Metot}

\subsection{Fiber Optik Kayıp Mekanizmaları}

Bu bölüm fiber optik bağlantı kayıplarından olan, Fresnel yansıma kayıpları ile boyuna hizalama kayıplarını açıklamaktadır. e-ISSN: 2148-2683
Ayrıca bu bölümde, iki farklı kayıp mekanizmasının birleşimine dayanarak oluşturulan fiber optik yer değiştirme sensör FOYDS sisteminin matematiksel alt yapısı incelenmektedir.

Telekomünikasyonda istenmeyen fiber optik kayıpları, fiber optik sensör oluşturmada kontrollü bir şekilde kullanılarak algılayııı mekanizmalara dönüştürülebilmektedir. Bu kayıplardan iki fiber birbirine eklendiği zaman bağlaşım hatalarından dolayı birinci fiberdeki gücün tamamı ikinci fibere aktarılamaz. $\mathrm{Bu}$ bağlantılarda bağlaşım kayıpları çeşitli sebeplerden ötürü oluşabilmektedir. Bunlar, Fresnel yansıma kayıpları ve fiber hizalama kayıpları olarak söylenebilir. Hizalama kayıpları ise üç durumdan oluşur bunlar: fiberler arasındaki ayrım (boyuna yanlış hizalama (longitudinal misalignment)), fiber öz eksenlerindeki dik kayma (yanal yanlış hizalama (lateral/radial/axial misalignment)) ve fiber öz eksenleri arasındaki açısal kayma (açısal yanlış hizalama (angular misalignment)). Şekil 1'de birinci fiberden gelen gücün bir kısmı yansıyarak geriye kalan kısmı ikinci fiberde kılavuzlanabilmektedir.

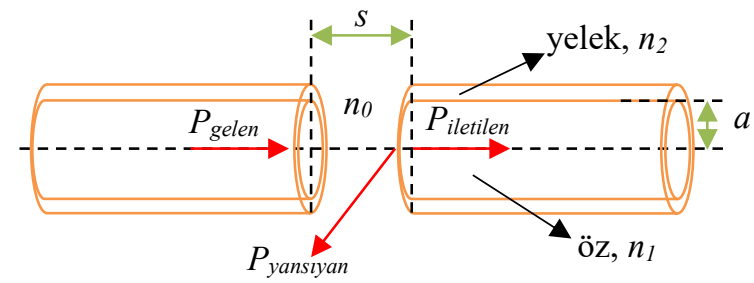

Şekil 1. Fiberde kayıp mekanizmaları.

Yansımadan ötürü kaynaklanan kayıp miktarını anlamak için öncelikle fiber-hava-fiber ara-yüzeylerinde oluşan yansıma katsayısını tanımlamak gerekir. $\mathrm{Bu}$ değer Eşitlik (1)'de verilmektedir [14].

$$
R=\frac{P_{\text {yanslyan }}}{P_{\text {gelen }}}=\left(\frac{n_{1}-n_{0}}{n_{1}+n_{0}}\right)^{2}
$$

Burada $P_{\text {gelen }}$ ve $P_{\text {yansiyan }}$ sirasıyla gelen ve yansıyan optik güçleri göstermektedir. $n_{l}$ ve $n_{0}$ ise sırasıyla fiber öz ve dış ortamın kırılma indis değerlerini temsil etmektedir. Buna bağlı olarak gelen ve iletilen güçler arasında da aşağıdaki gibi bir ilişki vardir.

$$
P_{\text {iletilen }}=(1-R) P_{\text {gelen }}
$$

Toplam yansıma kaybı, $L_{R} \mathrm{~dB}$ cinsinden Eşitlik (3) ile verilir [15].

$$
L_{R}=-10 \log (1-R)^{2}
$$

Şekil 1'deki gibi aralarında $s$ kadar bir açıklık bulunan fiber düzeneğindeki boyuna yanlış hizalamadan kaynaklı kuplaj kaybı (zayıflama), $L_{B}$ Eşitlik (4)'de verilmektedir [16].

$$
L_{B}=-10 \log \left(1-\frac{s \times N A}{3 \times n_{0} \times a}\right)
$$

Buradaki $a$ fiber öz yarıçapını ve $N A$ ise fiberin nümerik açıklık değerini ifade eder ve $N A$ değeri Eşitlik (5) ile 
hesaplanabilir [17]. Eşitlikteki $n_{2}$ fiber yelek kırılma indisini göstermektedir.

$$
N A=\sqrt{n_{1}^{2}-n_{2}^{2}}
$$

Toplam kayıp miktarı ise $\mathrm{dB}$ cinsinden aşağıdaki gibi verilir.

$$
L_{T}=L_{R}+L_{B}
$$

Buradan Eşitlik (3) ve (4) Eşitlik (6)'da yerine yazılırsa,

$L_{T}=-10 \log (1-R)^{2}-10 \log \left(1-\frac{s \times N A}{3 \times n_{0} \times a}\right)$

Eşitlik (7) sadeleştirildiğinde,

$$
L_{T}=-10 \log \left[(1-R)^{2} \times\left(1-\frac{s \times N A}{3 \times n_{0} \times a}\right)\right], \mathrm{dB}
$$

şeklinde elde edilir. Fiber kayıpları ile gelen/iletilen güçler arasındaki ilişki,

$$
L_{T}=10 \log \left(\frac{P_{\text {gelen }}}{P_{\text {iletilen }}}\right)
$$

ile verilmektedir [16]. Buradan iletilen güç yani fiber çıkışındaki güç Eşitlik (10)'daki gibi elde edilir.

$$
P_{\text {iletilen }}=P_{\text {gelen }} \times 10^{-\frac{L_{T}}{10}}
$$

\section{Araştırma Sonuçları ve Tartışma}

\subsection{Yer Değiştirmeye Göre Kayıp Değerleri}

İlk olarak $n_{l}=1.49, N A=0.51$ ve $a=490 \mu \mathrm{m}$ değerine sahip tipik bir PMMA plastik optik fiber için (OMC-FDPF4002EH) $n_{0}=1$ (hava) olarak seçildiğinde iki fiber arasındaki boyuna mesafeye $(s)$ göre, $L_{B}$ ve $L_{T}$ kayıp değerleri Şekil 2'de verilmiştir. Bu değerler için Eşitlik (3)'den $L_{R}=0,3430 \mathrm{~dB}$ bulunur. Şekil 2'ye bakıldığında iki fiber arasındaki mesafe arttıkça fiber boyuna hizalama kaybı artar ve sabit bir değere eşit olan fiber yansıma kayıpları da bu kayıp değerine eklenerek toplam kayıp miktarını oluşturur. Bu durumda sensör çıkışında optik güç azalacaktır.

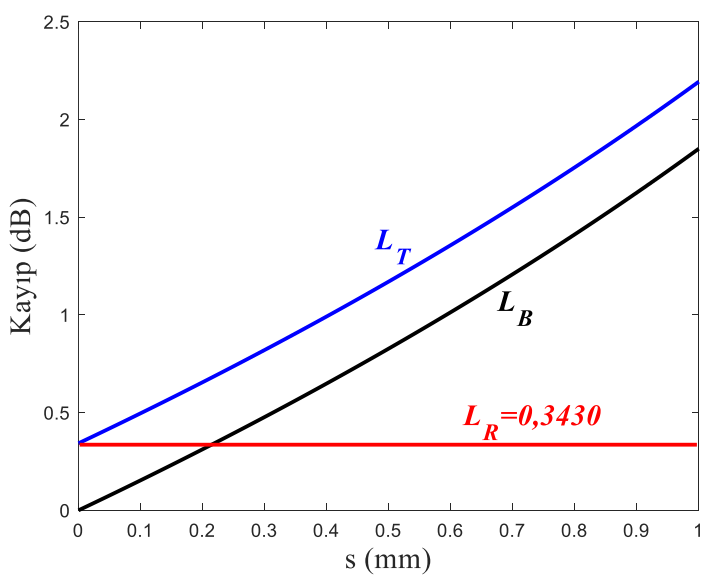

Şekil 2. Yer değiştirme miktarına göre fiber kayıpları.
İkinci durum olarak aynı fiber tipinin 1960/2000 $\mu \mathrm{m}$, 2944/3000 $\mu \mathrm{m}$ öz/yelek çap değerlerine sahip olan ve parça numaralar1 sirasiyla OMPF2000 ve OMPF3000 olan fiberler seçildiğinde yer değiştirme miktarına göre fiber kayıpları sırasıyla Şekil 3 (a) ve (b)'deki gibi olmaktadır. Şekil 2'den farklı olarak fiber öz yarıçapları arttığında kayıp miktarları azalmaktadır. Ve buna bağlı olarak maksimum yer değiştirme miktarını veren Eşitlik (11)'e göre ölçülebilecek en uzun mesafe değeri artarken sensör hassasiyetinde azalma olmaktadır. $s_{\text {limit }}$ değerine ulaştığında Eşitlik (8) değeri sonsuza yaklaşacak ve buna bağlı olarak Eşitlik (10) ile verilen çıkış gücü sıfır değerine eşit olacaktır.

$$
s_{\text {limit }}=\frac{3 \times n_{0} \times a}{N A}
$$

$980 \mu \mathrm{m}, 1960 \mu \mathrm{m}$ ve $2944 \mu \mathrm{m}$ öz çap değerlerine sahip olan bu üç fiber tipi için $s_{\text {limit }}$ değerleri sırasıyla, $2,9 \mathrm{~mm}, 5,8 \mathrm{~mm}$ ve $8,7 \mathrm{~mm}$ olarak hesaplanır. $1 \mathrm{~mm}$ altında ölçüm yapmak için 980/1000 (öz/yelek) $\mu \mathrm{m}$ çap değerine sahip olan fiber plastik optik fiber tipleri içerisinde en uygun fiber tipini temsil etmektedir.
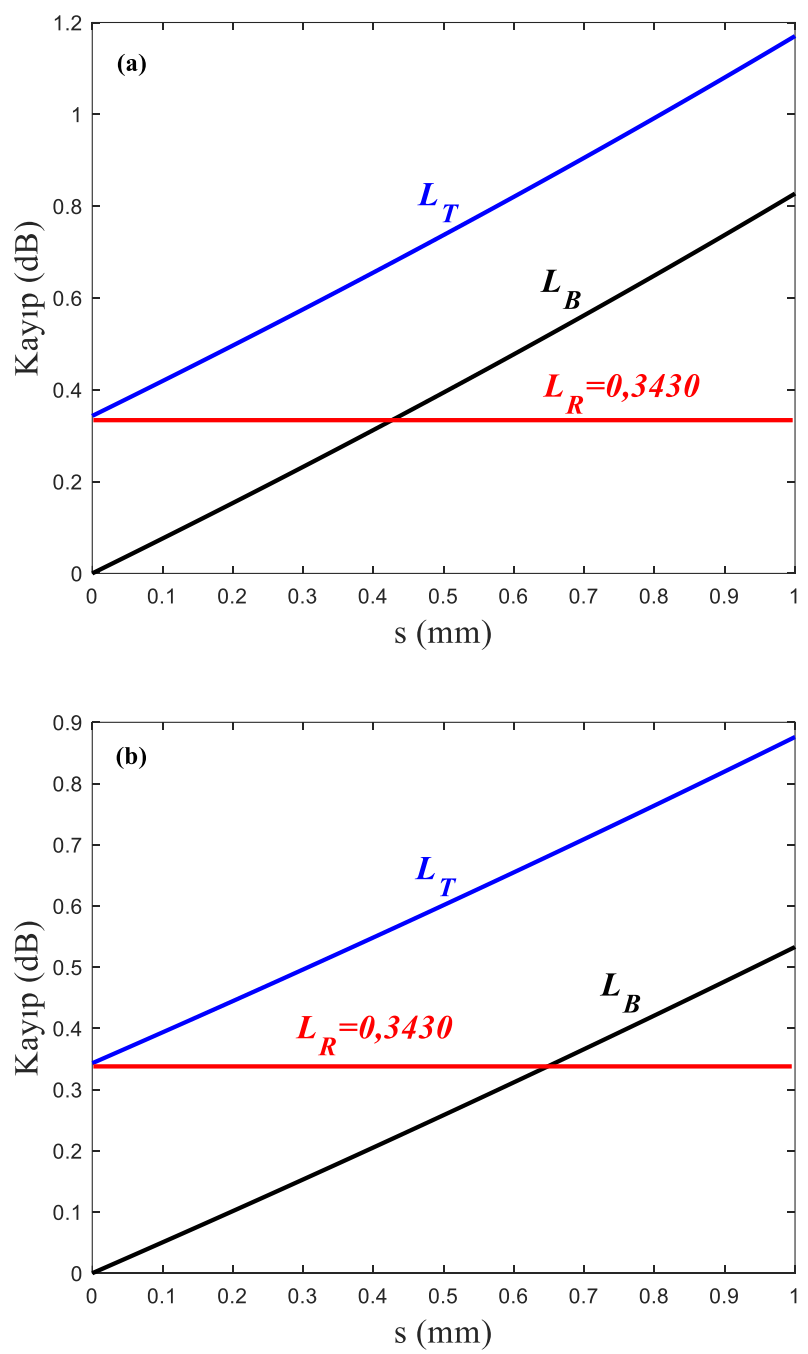

Şekil 3. Yer değiştirme miktarına göre fiber kayıpları.

\subsection{Yer Değiştirmeye Göre Sensör Çıkış Gücü}

$P_{\text {gelen }}$ güç $100 \mu \mathrm{W}$ olarak seçildiğinde çıkış gücü $\left(P_{\text {iletilen }}\right)$ mesafeye bağlı olarak üç fiber tipi için Şekil 3 'te gösterilmektedir. 


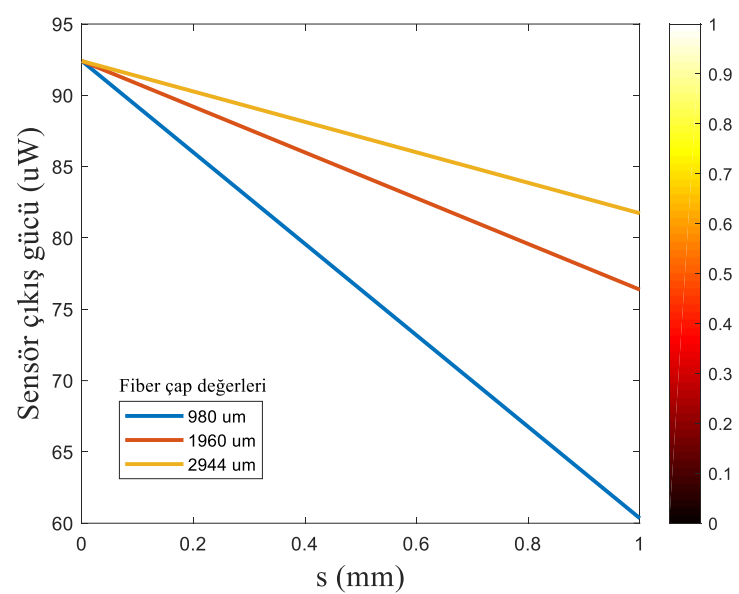

Şekil 3. Yer değiştirme miktarına göre sensör çıkış gücü $\left(P_{\text {gelen }}=100 \mu \mathrm{m}\right)$.

Bu çalışmada, Eşitlik (12) önerilen sensörün hassasiyetini (H) belirlemek için kullanılmıştır.

$$
H=\frac{\Delta P_{\text {iletilen }}}{\Delta s}(\mu \mathrm{W} / \mathrm{mm})
$$

Buna göre üç PMMA fiber tipi için $H$ değerleri Tablo 1 de verilmiştir.

Tablo 1. PMMA fiber tipleri için H değerleri.

\begin{tabular}{c|c}
\hline $\begin{array}{c}\text { Fiber öz çap değerleri, } \boldsymbol{2} \boldsymbol{a} \\
(\boldsymbol{\mu} \mathbf{m})\end{array}$ & $\begin{array}{c}\text { Hassasiyetlik, } \boldsymbol{H} \\
(\boldsymbol{\mu W} / \mathbf{m m})\end{array}$ \\
\hline 980 & 32,05 \\
\hline 1960 & 16,02 \\
\hline 2944 & 10,67 \\
\hline
\end{tabular}

\section{Sonuç}

Plastik optik fiberle oluşturulacak milimetre altı yer değiştirmenin algılanması için bir sensör modeli geliştirilmiştir. Sensör için fiber kayıp mekanizmalarından olan yansıma ve boyuna yanlış hizalama kayıplarından faydalanılmıştır. Buna göre oluşturulmuş olan sensörün matematiksel modeli ortaya konmuştur. Modelin cevabını incelemek için piyasada bulunan PMMA tabanlı tipik plastik optik fiber değerleri kullanılmıştır. Bu doğrultuda farklı çap değerlerine sahip olan fiber tipleri için 1 mm'nin altında yer değiştirme ölçümlerinde kullanılacak en uygun fiber tipinin 980/1000 (öz/yelek) $\mu \mathrm{m}$ çapa sahip olan fiber olduğu belirlenmiştir. Belirlenen bu fiberin hassasiyetlik derecesi giriş fiberine uygulanan güç $100 \mu \mathrm{W}$ olduğunda $32,05 \mu \mathrm{W} / \mathrm{mm}$ olarak bulunmuştur.

\section{Teşekkür}

Yazarlar, Erciyes Üniversitesi Klinik Mühendisliği Araştırma ve Uygulama Merkezi'ne, araştırma faaliyetlerine destekleri için teşekkür eder.

\section{Kaynakça}

[1] Berkovic, G., \& Shafir, E. (2012). Optical methods for distance and displacement measurements. Advances in Optics and Photonics, 4(4), 441-471.
[2] Werneck, M. M., \& Allil, R. C. S. (Eds.). (2019). Plastic Optical Fiber Sensors: Science, Technology and Applications. CRC Press.

[3] Gangopadhyay, T. K. (2004). Prospects for fibre Bragg gratings and Fabry-Perot interferometers in fibre-optic vibration sensing. Sensors and Actuators A: Physical, 113(1), 20-38.

[4] Saracoglu, O. G., \& Hayber, S. E. (2016). Bent fiber sensor for preservative detection in milk. Sensors, 16(12), 2094.

[5] Santos, J. L., \& Farahi, F. (Eds.). (2014). Handbook of optical sensors. Crc Press.

[6] Udd, E., Spillman Jr, W. B., 2011. Fiber Optic Sensors: An Introduction for Engineers and Scientists. John Wiley \& Sons, $498 \mathrm{pp}$.

[7] Chen, J. H., Huang, X. G., Zhao, J. R., Tao, J., He, W. X., \& Liu, S. H. (2010). Fabry-Perot interference-based fiber-optic sensor for small displacement measurement. Optics communications, 283(17), 3315-3319.

[8] Zhou, X., \& Yu, Q. (2010). Wide-range displacement sensor based on fiber-optic Fabry-Perot interferometer for subnanometer measurement. IEEE sensors journal, 11(7), 1602-1606.

[9] Wang, T., Zheng, S., \& Yang, Z. (1998). A high precision displacement sensor using a low-finesse fiber-optic FabryPérot interferometer. Sensors and Actuators A: Physical, 69(2), 134-138.

[10] Krishnan, G., Bidin, N., Abdullah, M., Ahmad, M. F. S., Bakar, M. A. A., \& Yasin, M. (2016). Liquid refractometer based mirrorless fiber optic displacement sensor. Sensors and Actuators A: Physical, 247, 227-233.

[11] Mehta, A., Mohammed, W., \& Johnson, E. G. (2003). Multimode interference-based fiber-optic displacement sensor. IEEE Photonics Technology Letters, 15(8), 11291131.

[12] Rahman, H. A., Harun, S. W., Saidin, N., Yasin, M., \& Ahmad, H. (2011). Fiber optic displacement sensor for temperature measurement. IEEE Sensors Journal, 12(5), 1361-1364.

[13] Trudel, V., \& St-Amant, Y. (2009). One-dimensional singlemode fiber-optic displacement sensors for submillimeter measurements. Applied optics, 48(26), 4851-4857.

[14] Shrivastav, A. M., Gunawardena, D. S., Liu, Z., \& Tam, H. Y. (2020). Microstructured optical fiber based Fabry-Pérot interferometer as a humidity sensor utilizing chitosan polymeric matrix for breath monitoring. Scientific reports, 10(1), 1-10.

[15] Özsoy, S. (2009). Fiber optik. Birsen Yayınevi.

[16] A. Weinert, Plastic Optical Fibers: Principles, Components, Installation. Berlin, Germany: Springer-Verlag, 1999, pp. $37-$ 45 .

[17] Bass, M., \& Van Stryland, E. W. (2002). Fiber Optics Handbook: fiber, devices, and systems for optical communications (No. Sirsi) i9780071386234). Optical Society of America. 